\title{
A Key Role for Neurotensin in Chronic-Stress-Induced Anxiety-Like Behavior in Rats
}

\author{
Catherine P Normandeau ${ }^{1,2}$, Ana Paula Ventura-Silva ${ }^{3,4}$, Emily R Hawken ${ }^{1,2}$, Staci Angelis ${ }^{1,2}$, Calvin Sjaarda ${ }^{5}$, \\ Xudong Liu ${ }^{5}$, José Miguel Pêgo ${ }^{3,4}$ and Éric C Dumont ${ }^{*, 1,2}$ \\ 'Center for Neuroscience Studies, Queen's University, Kingston, ON, Canada; ²Department of Biomedical and Molecular Sciences, Queen's \\ University, Kingston, ON, Canada; ${ }^{3}$ Life and Health Sciences Research Institute (ICVS), School of Health Sciences, University of Minho, Braga, \\ Portugal; ${ }^{4}$ ICVS/3B's__PT Government Associate Laboratory, Braga/Guimarães, Portugal; ${ }^{5}$ Queen's Genomics Lab at Ongwanada (QGLO), Queen's \\ University, Kingston, ON, Canada
}

\begin{abstract}
Chronic stress is a major cause of anxiety disorders that can be reliably modeled preclinically, providing insight into alternative therapeutic targets for this mental health illness. Neuropeptides have been targeted in the past to no avail possibly due to our lack of understanding of their role in pathological models. In this study we use a rat model of chronic stress-induced anxiety-like behaviors and hypothesized that neuropeptidergic modulation of synaptic transmission would be altered in the bed nucleus of the stria terminalis (BNST), a brain region suspected to contribute to anxiety disorders. We use brain slice neurophysiology and behavioral pharmacology to compare the role of locally released endogenous neuropeptides on synaptic transmission in the oval (ov) BNST of non-stressed (NS) or chronic unpredictably stressed (CUS) rats. We found that in NS rats, post-synaptic depolarization induced the release of vesicular neurotensin (NT) and corticotropin-releasing factor (CRF) that co-acted to increase ovBNST inhibitory synaptic transmission in 59\% of recorded neurons. CUS bolstered this potentiation (I00\% of recorded neurons) through an enhanced contribution of NT over CRF. In contrast, locally released opioid neuropeptides decreased ovBNST excitatory synaptic transmission in all recorded neurons, regardless of stress. Consistent with CUS-induced enhanced modulatory effects of NT, blockade of ovBNST NT receptors completely abolished stress-induced anxiety-like behaviors in the elevated plus maze paradigm. The role of NT has been largely unexplored in stress and our findings highlight its potential contribution to an important behavioral consequence of chronic stress, that is, exaggerated avoidance of open space in rats.

Neuropsychopharmacology (2018) 43, 285-293; doi: I0.1038/npp.2017.134; published online 16 August 2017
\end{abstract}

\section{INTRODUCTION}

While the stress response is integral for survival, prolonged exposure to stressors can have damaging consequences. Repeated exposure to aversive stressors predicts and contributes to mental illnesses such as generalized anxiety disorders, major depressive disorder, or post-traumatic stress disorder (Deppermann et al, 2014; Gosselin and Laberge, 2003; Hammen et al, 2009). However, the biochemical imbalances caused by repeated stress in the brain remain elusive and animal models of chronic stress are essential to elucidate these mechanisms (Conrad et al, 2011).

Repeated aversive stressors result in increased volume and dendritic branching as well as long-term alterations of excitatory synaptic transmission in the bed nucleus of the stria terminalis (BNST) (Conrad et al, 2011; Dabrowska et al, 2013; Glangetas et al, 2013; Hubert and Muly, 2014; McElligott et al, 2010; Pego et al, 2008; Vyas et al, 2003). Surprisingly, the

\footnotetext{
*Correspondence: Dr ÉC Dumont, Department of Biomedical and Molecular Sciences, Queen's University, II 6 Barrie Street, Biosciences Complex, Room 1445, Kingston, ON, Canada K7L 3N6, Tel: +613 533 699।, E-mail: eric.dumont@queensu.ca

Received 21 April 2017; revised I5 June 2017; accepted I6 June 2017; accepted article preview online 26 June 2017
}

effects of chronic stress on local $\gamma$-aminobutyric acid (GABA) transmission, imperative for fine-tuning neuronal output, have been largely unexplored in the BNST. Neuropeptides are potent modulators of GABA transmission in the BNST, but whether their function is altered in chronically stressed rats has never been investigated (Crowley et al, 2016; Kash and Winder, 2006; Krawczyk et al, 2013). Neuropeptides in the BNST may be affected by chronic stress due to their involvement in the modulation of stress- or aversion-related phenomena (Lezak et al, 2014; Walker et al, 2009).

Specifically, the oval nucleus of the BNST (ovBNST) contains high concentrations of many different neuropeptides and activation of this specific nucleus increases anxietylike behaviors suggesting it may be sensitive to chronic stress (Kim et al, 2013). Therefore, we hypothesized that chronic stress would change neuropeptide modulation of synaptic transmission in the ovBNST. We used the chronic unpredictable stress (CUS) paradigm to test this hypothesis, a preclinical model that mimics everyday stressors and invariably increases anxiety-like behaviors in rats (Cerqueira et al, 2007). Interestingly, the neuromodulatory effects of neurotensin (NT), but not of corticotropinreleasing factor (CRF), became sensitized after 1-month exposure to CUS. Accordingly, in vivo pharmacological 
blockade of ovBNST NT receptors (NTRs) had an anxiolytic effect in CUS rats. The neuropeptide NT is therefore a significant contributor to ovBNST promotion of anxiety-like behavior in chronically stressed animals.

\section{MATERIALS AND METHODS}

\section{Rats}

A total of 133 adult male rats (Charles River Laboratories, Canada/Spain) weighing 350-450 g were included in the electrophysiology experiments (Wistar and Long Evans rats, $n=63$ ) and behavioral experiments (Wistar rats, $n=70$ ). The rats were maintained on an artificial $12 \mathrm{~h}$ light/dark cycle (0800 hours lights on/2000 hours lights off) or $12 \mathrm{~h}$ dark/light cycle (0800 hours lights off/2000 hours lights on).

The rats acclimatized for a minimum of 1 week upon arrival to the facility. Rat chow and water were provided ad libitum in the home cages. In all, 63 rats were used for electrophysiology (Canada), 33 rats performed the elevated plus maze (EPM; Portugal), and 37 rats performed the EPM and the forced swim test (FST; Canada). All experiments were conducted in accordance with the guidelines from the Canadian Council on Animal Care in Science and approved by the Queen's University Animal Care Committee and with Portugal local regulations (European Union Directive 86/609/EEC).

\section{Slice Preparation and Electrophysiology}

Rats were anesthetized with isoflurane (5\% at $51 / \mathrm{min}$ ) and their brains removed into ice-cold artificial cerebral spinal fluid (aCSF) containing (in $\mathrm{mM}$ ): $126 \mathrm{NaCl} ; 2.5 \mathrm{KCl} ; 1.2$ $\mathrm{MgCl}_{2} ; 6 \mathrm{CaCl}_{2} ; 1.2 \mathrm{NaH} 2 \mathrm{PO}_{4} ; 25 \mathrm{NaHCO}_{3} ;$ and 12.5 D-glucose equilibrated with $95 \% \mathrm{O}_{2} / 5 \% \mathrm{CO}_{2}$. Brains were cut in $2{ }^{\circ} \mathrm{C}$ aCSF into coronal slices $(250 \mu \mathrm{m})$ with a vibrating blade microtome (VT-1000; Leica). We used the slice corresponding to $-0.26 \mathrm{~mm}$ from bregma. Slices were incubated at $34^{\circ} \mathrm{C}$ for $60 \mathrm{~min}$ and transferred to a chamber perfused $(3 \mathrm{ml} / \mathrm{min})$ with aCSF at $34^{\circ} \mathrm{C}$. Remaining slices were kept in aCSF at room temperature until further use. Whole-cell voltage-clamp recordings were made using glass microelectrodes (3-5 M 2 ) filled with (in $\mathrm{mM}$ ): $70 \mathrm{~K}$ +-gluconate; $80 \mathrm{KCl} ; 1$ EGTA; 5 HEPES; 2 MgATP; 0.3 GTP; and 1 P-creatine. We recorded lateral from an imaginary line drawn vertically across the lateral ventricle and medial to the internal capsule. In the dorso-ventral plan, we only recorded dorsally to an imaginary horizontal line drawn halfway between the ventral tip of the lateral ventricle and the top of the anterior commissure as illustrated in our previous publications (Krawczyk et al, 2011a; Krawczyk et al, 2011b). Paired electrical stimuli (10-100 $\mu \mathrm{A}, 0.1 \mathrm{~ms}$ duration, $20 \mathrm{~Hz}$ ) were applied at $0.1 \mathrm{~Hz}$. Excitatory or inhibitory postsynaptic currents (E/IPSCs) were evoked by local fiber stimulation with tungsten bipolar electrodes while neurons were voltage-clamped at $-70 \mathrm{mV}$. GABA $\mathrm{A}_{\mathrm{A}}$-IPSC and AMPAEPSC were pharmacologically isolated with 6,7-dinitroquinoxaline-2,3-dione (DNQX; $50 \mu \mathrm{M})$ or picrotoxin $(100 \mu \mathrm{M})$, respectively. To induce local endogenous neuropeptide release, post-synaptic neurons were repetitively depolarized in voltage clamp from -70 to $0 \mathrm{mV}(100 \mathrm{~ms})$ at a frequency of $2 \mathrm{~Hz}$ for $5 \mathrm{~min}$ (Iremonger and Bains, 2009).
We defined long-lasting post-synaptic depolarizationinduced changes in E or IPSC peak amplitude as a $>20 \%$ deviation from baseline, $25 \mathrm{~min}$ following the end of the repetitive depolarization protocol. Recordings were made using a Multiclamp 700B amplifier and a Digidata 1440A (Molecular Devices Scientific). Data were acquired and analyzed with Axograph X running on Apple computers.

\section{Drugs}

Stock solutions of SR $142948(10 \mathrm{mM})$ and naloxone (Nal; $1 \mathrm{mM}$ ) were prepared in double-distilled water, and stock solutions of DNQX (100 mM), NBI-27914 (50 mM), and concanamycin A $(1 \mathrm{mM})$ were prepared in DMSO (100\%). All drugs were further dissolved in the physiological solutions or $0.9 \%$ saline at the desired concentrations (DNQX $50 \mu \mathrm{M}$, SR-142948 5-10 $\mu \mathrm{M}$, NBI-27914 $1 \mu \mathrm{M}$, concanamycin A $5 \mu \mathrm{M}$, and $\mathrm{Nal} 1 \mu \mathrm{M}$ ) and the final DMSO concentration never exceeded $0.1 \%$. Drugs were obtained from Sigma-Aldrich or R\&D Systems.

\section{Chronic Unpredictable Stress}

Rats were singly housed and randomly assigned to nonstressed (NS) or CUS groups. Rats in the NS group were handled regularly over 4 weeks. Rats in the CUS group were exposed to 4 weeks of daily exposure to one stressor (10-60 $\mathrm{min} /$ day) at different times, as described previously (Cerqueira et al, 2007). Stressors presentation was randomized and included one of the following aversive stimuli: cold water immersion $\left(18^{\circ} \mathrm{C}, 60 \mathrm{~min}\right)$, home cage shaking (10 $\mathrm{min})$, restraining (60 $\mathrm{min})$, overcrowding (3-4 rats/cage, $60 \mathrm{~min})$, and exposure to hot air stream $(15 \mathrm{~min})$.

\section{Surgery}

Rats were positioned in a stereotaxic instrument and secured by non-rupture ear bars under isoflurane $(2-3 \%, 51 / \mathrm{min})$ or ketamine/medetomidine anesthesia. Double-guide cannulas (Plastics One) were bilaterally implanted $1 \mathrm{~mm}$ above the upper limit of the oval region of the dorsal BNST (dBNST; $-0.26 \mathrm{AP}, \pm 1.9 \mathrm{ML}$, and $-6.5 \mathrm{DV})$. Injector cannulas (Plastics One) were placed into the guide cannulas $(7.5 \mathrm{~mm}$ length). All stereotaxic coordinates were relative to bregma. The head attachment was secured in place via four $0.08 \times 0.125$ in jeweler screws and dental acrylic cement. The guide cannulas were fitted with an autoclaved $30 \mathrm{Ga}$ stylet and covered with a screw-on dust cap. Following surgery, the rats recovered for 1 week and then were randomly assigned to six experimental groups: NS (saline, $n=11)$; NS SR 5 (SR-142948 $5 \mu \mathrm{M}, n=8)$; NS SR 10 (SR-142948 $10 \mu \mathrm{M}, n=6$ ); CUS saline (saline, $n=16$ ); CUS SR 5 (SR-142948 $5 \mu \mathrm{M}, n=12$ ); and CUS SR 10 (SR 142948 $10 \mu \mathrm{M}, n=17$ ). Three rats (two in the NS SR5 group and one in the NS SR 10 group) were killed before the FST due to health issues.

\section{Behavioral Tests}

The rats were placed in the NS groups or the CUS groups receiving a $300 \mathrm{nl}$ injection of either saline, SR-142948 $5 \mu \mathrm{M}$, or SR-142948 $10 \mu \mathrm{M} 30 \mathrm{~min}$ before testing (Binder et al, 
2001). Behavioral testing was done on 3 consecutive days, starting with the EPM followed by the FST.

\section{Elevated Plus Maze}

Rats were tested for $5 \mathrm{~min}$ in the EPM using a black polypropylene 'plus'-shaped maze (Med Associates) as previously described (Pego et al, 2008). The maze consisted of two facing open arms $(50.8 \times 10.2 \mathrm{~cm})$ and two closed arms $(50.8 \times 10.2 \times 40.6 \mathrm{~cm}), 72 \mathrm{~cm}$ above the floor. Testing was performed under bright white light ( $\cong 40$ lux). The time spent in the open arms, junction area, and closed arms, as well as the number of entrances and explorations in each section were recorded using a system of infrared photo beams, the crossings of which were monitored by a computer. The times spent in each of the compartments of the EPM are presented as percentage of the total duration of the trial.

\section{Forced Swim Test}

Rats were introduced to a cylindrical container filled with $30 \mathrm{~cm}$ of water $\left(23-25^{\circ} \mathrm{C}\right)$ for $15 \mathrm{~min}$ during pretest and 5 min during testing. The rats' behavior was categorized as (1) immobile, (2) swimming, and (3) climbing (included diving). We defined immobile as the absence of directed movements, climbing as vertical movement of the forepaws and swimming as horizontal movement in the swim chamber. The predominant behavior over each $5 \mathrm{~s}$ period of the $300 \mathrm{~s}$ test was rated over a total score of 60 by an experimenter blind to the pharmacological treatment or the stress group.

\section{Open Field}

Animals were individually tested for $5 \mathrm{~min}$ each in an open field $(\mathrm{OF})$ arena $(43.2 \times 43.2 \mathrm{~cm})$ that had transparent acrylic walls and a white floor (model ENV-515, MedAssociates, St Albans, VT 05478). Each subject was initially placed in the center of the arena and horizontal activity and instant position were registered, using a system of two 16-beam infrared arrays connected to a computer. Total distances were used as indicators of locomotor activity.

\section{Histological Procedures}

Following behavioral testing, the rats were anesthetized with pentobarbital or isoflurane. Extracted brains were submerged in fresh paraformaldehyde for 2 days and switched to $30 \%$ sucrose paraformaldehyde for cryoprotection. The brains were kept at $-80^{\circ} \mathrm{C}$ until histology. The $30 \mu \mathrm{m}$ coronal sections were sliced and stained with cresyl violet to assess the location of the central injections (Figure $5 b$ and $c$ ).

\section{RNA Extraction and Reverse Transcription}

Brain sections containing the dBNST or the central amygdale (CeA) (Supplementary Figure S1) were collected from RNAlater (ThermoFisher Scientific) solution with a sterile tissue puncher and submerged in $100 \mu \mathrm{l}$ of lysis/binding buffer from the Dynabeads mRNA Direct Micro Kit (ThermoFisher Scientific). The tissue was immediately homogenized in microcentrifuge tubes using a disposable pestle (Fisherbrand). mRNA was purified using the Dynabeads mRNA Direct Micro Kit (ThermoFisher Scientific) following the manufacturer's recommended protocol for mRNA isolation from tissues. mRNA concentration was determined using the Qubit Fluorometer 2.0 (ThermoFisher Scientific). A unit of $40 \mathrm{ng}$ of mRNA from each sample was reverse transcribed using the SuperScript IV First-Strand Synthesis kit (Invitrogen) in an Applied Biosystems GeneAmp PCR System 9700 (ThermoFisher Scientific).

\section{Real-Time qPCR and Data Analysis}

The cDNA was amplified in the ViiA7 Real-Time PCR machine (ThermoFisher Scientific) with a two-step PCR protocol $\left(95^{\circ} \mathrm{C}\right.$ for $10 \mathrm{~min}$ followed by 40 cycles of $95^{\circ} \mathrm{C}$ for $15 \mathrm{~s}$ and $60^{\circ} \mathrm{C}$ for $\left.1 \mathrm{~min}\right)$ using the Power SYBR Green PCR Master Mix (ThermoFisher Scientific) and KiCqStart SYBR Green Primers (Sigma-Aldrich) (Supplementary Table S1). Each reaction was performed in triplicate and dissociation curves were generated for all reactions to ensure primer specificity. All target genes were normalized to three reference genes (Sdha, Actb, and Hprt) and the relative quantification using the comparative $\mathrm{Ct}$ method was determined using the DataAssist Software Version 3.01 (ThermoFisher Scientific).

\section{Statistical Analyses}

Changes in E/IPSC peak amplitude were measured from baseline and are shown as percentages as follows: (peak amplitude post $_{-}$ peak amplitude baseline/peak amplitude $\left._{\text {baseline }}\right) \times 100$. Data are reported as means $\pm S E M$ and each data point represents the average of values in $1 \mathrm{~min}$ bins (six evoked E/IPSCs) across recorded neurons.

Two-way ANOVAs were used to compare multiple means of parametric data and Kruskal-Wallis $H$-test for nonparametric. A Bonferroni correction was used for multiple comparisons. Mann-Whitney $U$-test was used to compare specific means with an adjusted $p$-value according to the number of test performed. Fisher's exact tests and $\chi^{2}$ analyzed contingency tables of the neuronal response distribution. All statistical analyses were done with SPSS Statistics Version 23 (SAS Institute) or Prism 6.

\section{RESULTS}

Post-synaptic activation of ovBNST neurons $(0 \mathrm{mV}, 100 \mathrm{~ms}$, $2 \mathrm{~Hz}, 5 \mathrm{~min}$ ) resulted in robust long-lasting depolarizationinduced enhancement of inhibition (l-DEI) in $59 \%$ of recorded neurons (time $\times$ group, $\mathrm{F}_{1,32}=7.9, \quad p<0.0001$, $n=20 / 34$ cells 1 -DEI from 21 rats; Figure $1 \mathrm{a}$ and $\mathrm{f}$ ). The addition of the v-ATPase inhibitor concanamycin A $(5 \mu \mathrm{M})$ to the intracellular recording solution completely ablated ovBNST 1-DEI that was thus vesicular release-dependent (Fisher's exact test (NS-aCSF vs NS-Conc), $p=0.04, n=0 / 4$ cells 1-DEI from 2 rats; Figure $1 \mathrm{~b}$ and $\mathrm{f}$ ). In addition, rat strain and light cycle had no effect on 1-DEI cell response $\left(\chi X^{2}{ }_{(2, n=34)}=4.69, p=0.1\right.$; Supplementary Table S2).

The ovBNST is exclusively populated with GABA neurons that also contain the neuropeptides NT, CRF, dynorphin, or enkephalin (Day et al, 1999; Ju et al, 1989b; Poulin et al, 


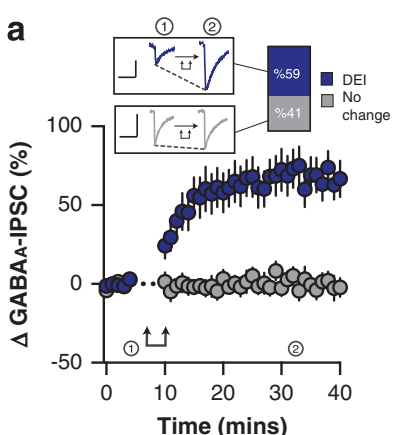

b

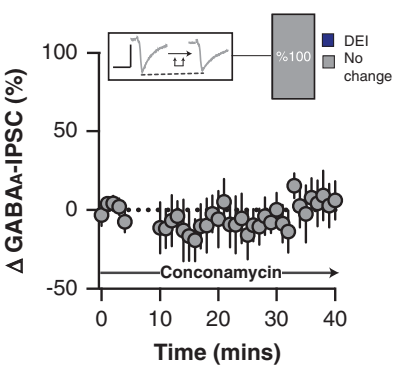

C

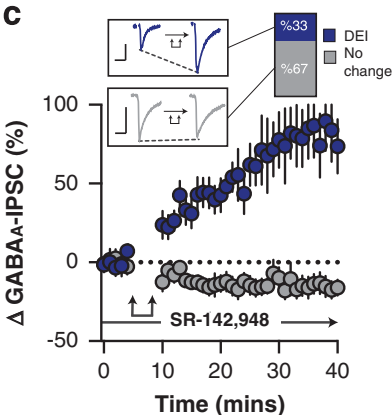

d
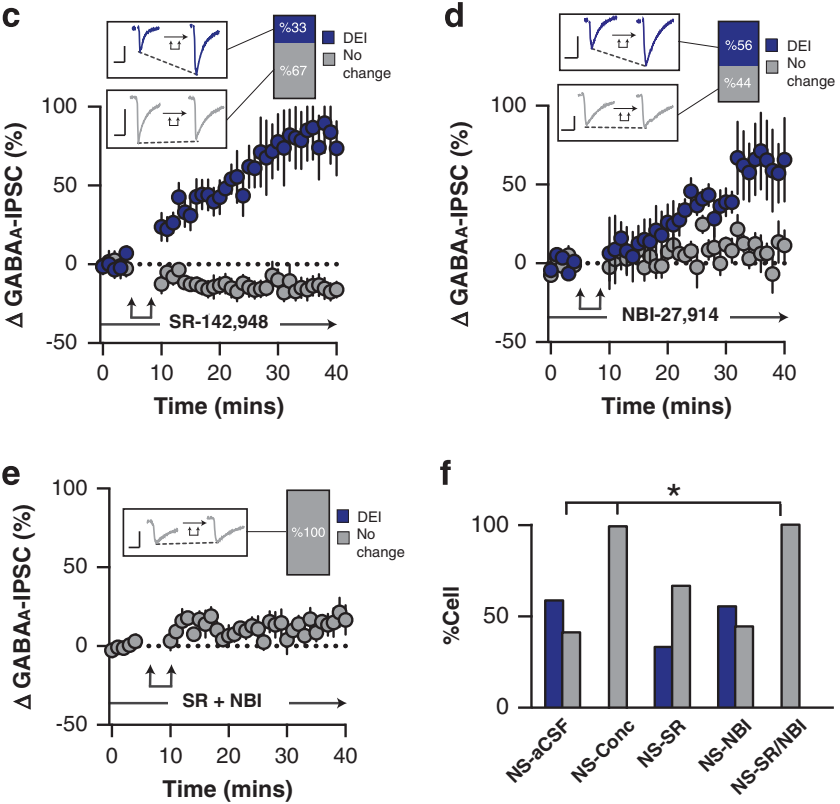

f

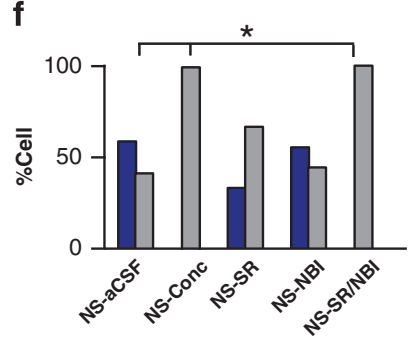

Figure I Effect of post-synaptic depolarization on $G_{A B A}$ synaptic transmission in the ovBNST of non-stressed (NS) rats. Effect of postsynaptic activation on the amplitude of electrically evoked GABA $A_{A}-I P S C S$ over time in the ovBNST of NS rats in (a) aCSF, (b) with intracellular concanamycin $(5$ HM), (c) with extracellular SR-I42948 (I 0 HM), (d) with extracellular NBI-279|4, and (e) with extracellular NBI-279|4 (I HM) and SR-I42948 (I 0 MM). Insets in a-e show representative ovBNST GABA $A^{-}$ IPSCs before and after post-synaptic activation followed by the proportion of responding neurons. Scale bar: $250 \mathrm{pA}$ and $25 \mathrm{~ms}$. Double arrows represent post-synaptic depolarization ( $0 \mathrm{mV}, 100 \mathrm{~ms}$ at $2 \mathrm{~Hz}, 5 \mathrm{~min})$. (f) Histogram summarizing the proportion of responding neurons to postsynaptic depolarization across different pharmacological treatments. $* p<0.05$.

2009). NT and CRF both increase ovBNST GABA $A_{A}$-IPSCs through either NTR or CRF receptor 1 (CRFR1), respectively, and we hypothesized that one or both could be responsible for 1-DEI (Kash and Winder, 2006; Krawczyk et al, 2013). As such we used a non-selective NTR antagonist (SR-142948, $10 \mu \mathrm{M})$ and a CRFR1 selective antagonist (NBI-27914, $1 \mu \mathrm{M})$. Blocking NTR did not significantly block 1-DEI (Fisher's exact test (NS-aCSF vs NS-SR), $p=0.1$, $n=6 / 18$ cells 1 -DEI from 11 rats; Figure $1 \mathrm{c}$ and $\mathrm{f}$ ). Likewise, l-DEI was unaltered ( $56 \%$ of neurons) by the CRF antagonist (Fisher's exact test (NS-aCSF vs NS-NBI), $p=1.0, n=5 / 9$ cells l-DEI from 6 rats; Figure $1 \mathrm{~d}$ and f). However, co-application of SR-142948 and NBI-27914 completely eliminated 1-DEI indicating cooperation between NT and a
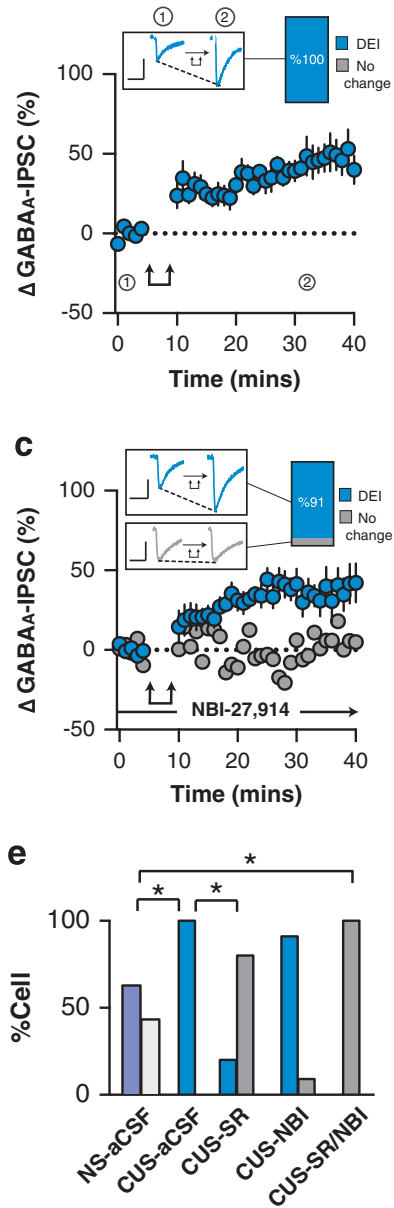
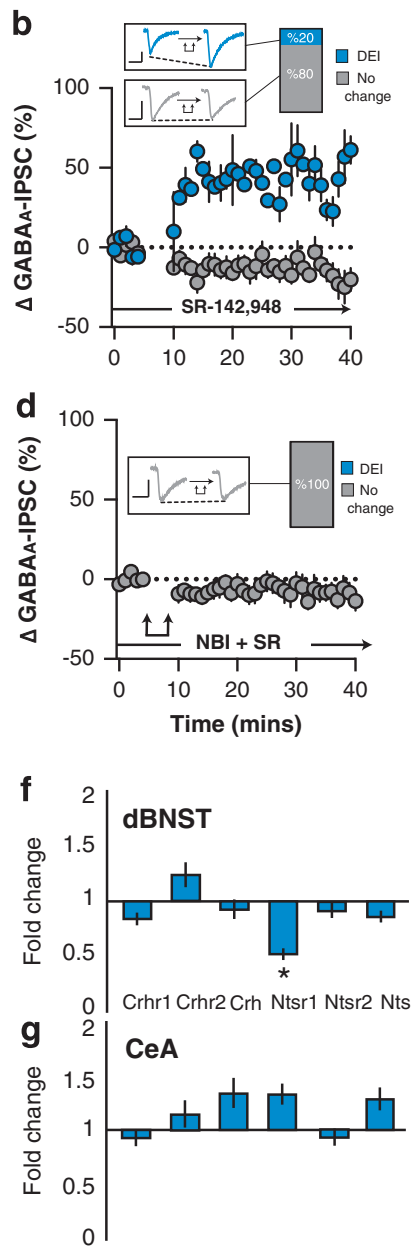

Figure 2 Effect of post-synaptic depolarization on $G A B A_{A}$ synaptic transmission in the ovBNST of chronic unpredictable stress (CUS)-exposed rats. Effect of post-synaptic activation on the amplitude of electrically evoked GABA $_{A}$-IPSCs over time in the ovBNST of CUS rats in (a) aCSF, (b) with

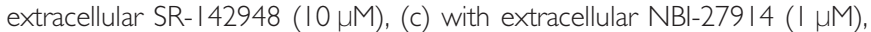
and (d) with extracellular NBI-279 I4 (I HM) and SR-I 42948 (I 0 HM). Insets in a- $d$ show representative ovBNST-evoked GABA $A_{A}$ IPSCs before and after post-synaptic activation followed by the proportion of responding neurons. Scale bar: $250 \mathrm{pA}$ and $25 \mathrm{~ms}$. Double arrows represent post-synaptic depolarization ( $0 \mathrm{mV}, 100 \mathrm{~ms}$ at $2 \mathrm{~Hz}, 5 \mathrm{~min}$ ). (e) Histogram summarizing the proportion of responding neurons to post-synaptic depolarization across different pharmacological treatments. (f, g) Histogram showing fold change in mRNA expression of Crhrl, Crhr2, Crh, Ntsrl, Ntsr2, and Nts in CUS compared to NS rats in the dBNST and CeA. $* p<0.05$.

CRF in producing l-DEI, which is consistent with their colocalization in ovBNST neurons (Fisher's exact test (NSaCSF vs NS-SR/NBI), $p=0.004, n=0 / 8$ cells 1 -DEI from 4 rats; Figure 1e and f) (Ju and Han, 1989a). Interestingly, bath application of NTR antagonist but not of CRFR1 antagonist resulted in a reversible depression of $\mathrm{GABA}_{\mathrm{A}}$-IPSCs suggesting constitutive NTR activity (Supplementary Figure S2). In addition, neither NT bath application or repetitive depolarization changed holding current or input resistance indicating membrane potential and channels were not changed by the neuropeptide (Supplementary Table S3).

We next determined whether 1-DEI might be altered in the ovBNST of chronically stressed rats. CUS significantly facilitated 1-DEI (time, $\mathrm{F}_{1,10}=5.0, p=0.009, n=11 / 11$ cells 
1-DEI from 7 animals; Figure 2a) that was now measurable in all tested neurons compared to the NS group $\left(\chi_{(1, n=45)}^{2}=6.6\right.$, $p=0.01$; Figure 2e). The NTR antagonist significantly reversed CUS-induced facilitation of 1-DEI suggesting that NT took over modulation of ovBNST inhibitory synaptic transmission in stressed conditions (Fisher's exact test (CUS-aCSF vs CUS-SR), $p=0.0002, n=2 / 10$ cells $1-\mathrm{DEI}$ from 4 rats; Figure $2 \mathrm{~b}$ and e). In contrast, CRFR1 blockade had no effect on 1-DEI in CUS rats (Fisher's exact test (CUS-aCSF vs CUS-NBI), $p=1.0, n=11 / 12$ cells l-DEI from 3 rats; Figure $2 \mathrm{c}$ and e) although both CRF and NT antagonists were necessary to completely eliminate 1-DEI (Fisher's exact test (CUS-aCSF vs CUS-SR/NBI), $p=0.0001$, $n=0 / 8$ cells 1 -DEI from 5 rats; Figure $2 d$ and e).

We then investigated changes in mRNA expression of $\mathrm{CRF}, \mathrm{NT}$, and their receptors in the dBNST and, the CeA that has strong inhibitory inputs onto the ovBNST and a similar neuropeptide array (expressing both CRF and NT) (Day et al, 1999). In support of CUS-induced changes in the NT system, CUS significantly and selectively reduced dBNST Ntsr1 mRNA levels compared to NS $(p=0.05$; Figure 2f). In contrast, CUS had no significant effect on other stress-related transcripts in either the dBNST or the CeA (Figure $2 \mathrm{f}$ and $\mathrm{g}$ ).

In NS animals, post-synaptic depolarization resulted in long-lasting depolarization-induced reduction of excitatory synaptic transmission (l-DRE) in all tested neurons (time, $\mathrm{F}_{1,7}=12.2, p<0.0001, n=9 / 9$ cells 1 -DRE from 6 rats; Figure $3 \mathrm{a}$ and $\mathrm{c})$. The broad-spectrum opioid receptor antagonist Nal $(10 \mu \mathrm{M})$ abolished l-DRE suggesting that post-synaptic depolarization triggered the local release of endogenous opioids (Fisher's exact test (NS-aCSF vs NS-Nal), $p=0.002$, $n=2 / 9$ cells 1 -DRE from 4 rats; Figure $3 \mathrm{~b}$ and $\mathrm{c}$ ). The effect of post-synaptic activity on excitatory transmission was largely unaffected by CUS and still resulted in robust 1-DRE in the vast majority of recorded ovBNST neurons (time, $\mathrm{F}_{1,5}=4.2, p=0.05, n=6 / 7$ cells l-DRE from 3 rats; Fisher's exact test (NS $v s$ CUS), $p=0.4$; Figure $4 \mathrm{a}$ and c). Similar to NS conditions, Nal completely blocked l-DRE (Figure 4b), supporting the involvement of locally released endogenous opioids in this response (Fisher's exact test (CUS-aCSF vs
CUS-Nal), $p=0.005, n=0 / 6$ cells l-DRE from 3 rats; Figure 4c).

CUS increases avoidance of open arms in the EPM and immobilization in the FST (Bessa et al, 2009). Converging evidence suggests that the BNST has a key role in these chronic stress-induced anxiety- and depression-like phenomena (Daniel and Rainnie, 2016). As CUS altered the neuromodulatory effect of NT in the ovBNST, we hypothesized that in vivo pharmacological blockade of ovBNST NTR might reverse CUS-induced avoidance of open arms in the EPM and immobility in the FST. As expected, CUS significantly reduced the percentage of time spent in the open arms in saline-treated rats $(U=5$, $p=0.0002$; Figure 5d). Intra-ovBNST SR-142948 (5-10 $\mu \mathrm{M} /$ side) had no effect on EPM behaviors in NS but significantly increased the percentage of time spent in the open arms in CUS (Kruskal-Wallis $H$-test, $\chi_{5}^{2}=18.2, p=0.003$; Figure $5 \mathrm{~d}$ ). SR-142948 (5-10 $\mu \mathrm{M} /$ side) dose-dependently reversed this effect in CUS rats $(U=7, p=0.0001$; Figure $5 d)$. SR-142948 had no effect on the number of open arms entries (KruskalWallis $H$-test, $\chi^{2}{ }_{5}=5.3, p=0.4$; Figure 5e) and did not affect total distance traveled in the OF $\left(\mathrm{F}_{(3,32)}=0.7, p=0.6\right.$; Supplementary Figure S3) therefore did not affect locomotion. In our conditions, intra-ovBNST NTRs blockade had no effect on immobility scores in the FST in either NS or CUS conditions (Kruskal-Wallis $H$-test, $\chi^{2}{ }_{5}=8.6, p=0.1$; Figure 5f).

\section{DISCUSSION}

We used brain slice whole-cell voltage-clamp recordings and discovered that in the ovBNST of NS rats, post-synaptic activation resulted in long-lasting depolarization-induced enhancement of inhibitory $\mathrm{GABA}_{\mathrm{A}}$ and reduction of excitatory AMPA synaptic transmission that we termed 1-DEI and 1-DRE, respectively. NT and CRF both produced 1-DEI while opioids were fully responsible for 1-DRE. CUS facilitated 1-DEI through an enhanced contribution of NT, whereas 1-DRE was not affected. Pharmacological blockade of ovBNST NTRs abolished CUS-induced reduction in open arm avoidance in the EPM, suggesting that NT may
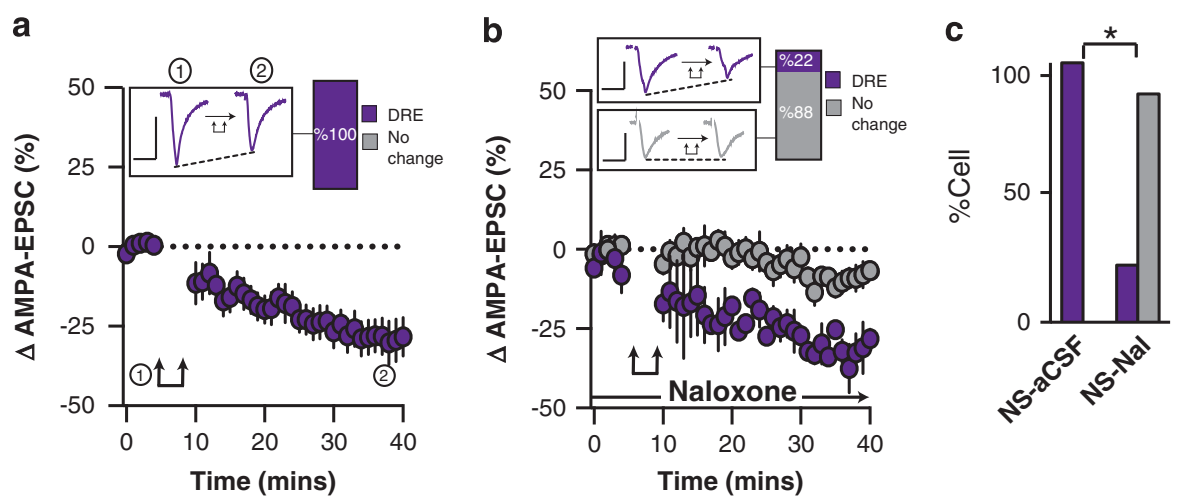

Figure 3 Effect of post-synaptic depolarization on AMPA synaptic transmission in the ovBNST of NS rats. Effect of post-synaptic activation on the amplitude of electrically evoked AMPA-EPSCs over time in the ovBNST of NS rats in (a) aCSF and (b) with extracellular naloxone (I HM). Insets in (a and b) show representative ovBNST-evoked AMPA-EPSCs before and after post-synaptic activation followed by the proportion of responding neurons. Scale bar: $250 \mathrm{pA}$ and $25 \mathrm{~ms}$. Double arrows represent post-synaptic depolarization $(0 \mathrm{mV}, 100 \mathrm{~ms}$ at $2 \mathrm{~Hz}, 5 \mathrm{~min})$. (c) Histogram summarizing the proportion of responding neurons to post-synaptic depolarization across different pharmacological treatments. $* p<0.05$. 

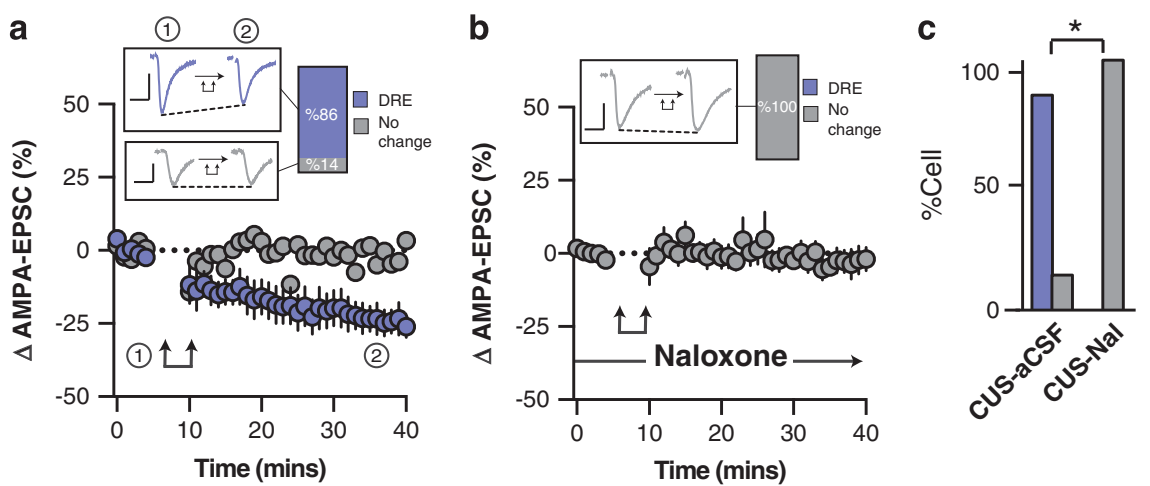

Figure 4 Effect of post-synaptic depolarization on AMPA synaptic transmission in the ovBNST of CUS rats. Effect of post-synaptic activation on the amplitude of electrically evoked AMPA-EPSCs over time in the ovBNST of CUS rats in (a) aCSF and (b) with extracellular naloxone (I HM). Insets in (a and b) show representative ovBNST-evoked AMPA-EPSCs before and after post-synaptic activation followed by the proportion of responding neurons. Scale bar: $250 \mathrm{pA}$ and $25 \mathrm{~ms}$. Double arrows represent post-synaptic depolarization ( $0 \mathrm{mV}, 100 \mathrm{~ms}$ at $2 \mathrm{~Hz}, 5 \mathrm{~min}$ ). (c) Histogram summarizing the proportion of responding neurons to post-synaptic depolarization across different pharmacological treatments. ${ }^{*} p<0.05$.

a

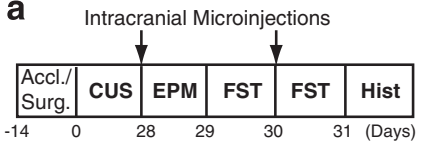

b

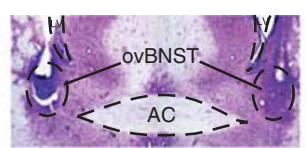

C

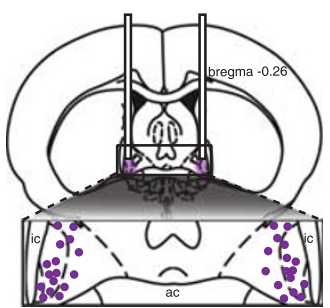

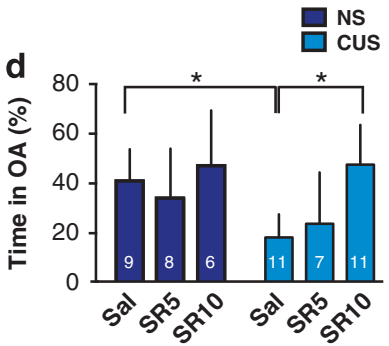

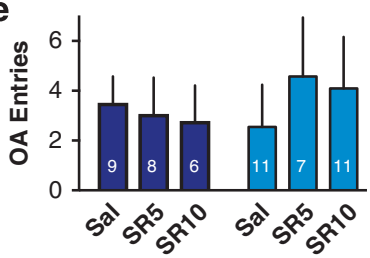

f

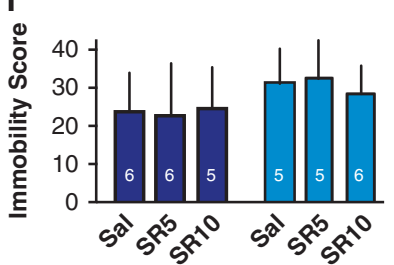

Figure 5 Effect of intra-ovBNST NTR pharmacological blockade on elevated plus maze (EPM) and forced swim test (FST) behaviors in NS and CUS rats. (a) Experimental timeline. (b) Photomicrograph showing a representative accurate bilateral cannula placement. (c) Drawing showing all intracranial cannula placements. (d) Bar graph representing the percentage of time spent in the EPM open arms (OA) across experimental groups. (e) Bar graph representing the number of OA entry across experimental groups. (f) Bar graph representing immobility scores in the FST across experimental groups. Accl./Surg., acclimatation/surgeries; Hist, histology; Sal, saline; SR5, SR

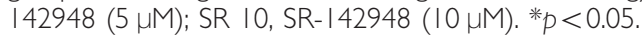

contribute to anxiety disorders (Laszlo et al, 2010; Saiz Ruiz et al, 1992).

In NS rats, post-synaptic activation produced l-DEI in slightly over half $(59 \%)$ of recorded ovBNST neurons. There is clear evidence of various ovBNST neuron subpopulations with distinct morphological, neurochemical, or electrophysiological signatures that could explain this dichotomy

(Day et al, 1999; Hammack et al, 2007; Ju et al, 1989b; Larriva-Sahd, 2006; Poulin et al, 2009). The neuron-specific expression of 1-DEI may be tightly linked with specific neuropeptidergic profiles (Iremonger and Bains, 2009; Ludwig and Pittman, 2003). NT and CRF are highly concentrated in ovBNST neurons and both neuropeptides robustly potentiate $\mathrm{GABA}_{\mathrm{A}}$-mediated synaptic transmission although through distinct pre- and post-synaptic loci, respectively (Day et al, 1999; Ju and Han, 1989a; Kash and Winder, 2006; Krawczyk et al, 2013). A study combining brain slice electrophysiology and single-cell PCR showed that $60 \%$ of ovBNST neurons contain CRF, which is precisely the percentage of 1-DEI response we obtained, supporting a role for CRF in l-DEI (Dabrowska et al, 2011). Importantly, CRF and NT colocalize in the ovBNST, and pharmacological blockade of both CRFR1 and NTR was necessary to completely abolish l-DEI (Ju and Han, 1989a). Application of either neuropeptide antagonist alone did not block l-DEI suggesting a cooperative mechanism, where one neuropeptide activity can compensate for the blockage of the other. The exact functional link however remains elusive.

Post-synaptic activation also resulted in opioid-dependent l-DRE in all recorded ovBNST neurons in NS rats, mitigating the possibility of subpopulation effects. Only $41 \%$ of ovBNST neurons seem to express detectable amounts of enkephalins mRNA, which poorly colocalizes with CRF or NT (Day et al, 1999). Dynorphin is also abundant in the rat ovBNST and may have contributed to the opioid-dependent l-DRE we measured (Poulin et al, 2009). Enkephalin and dynorphin are potent inhibitors of excitatory synaptic transmission in the brain, supporting opioid-dependent l-DRE (Crowley et al, 2016). Opioid neuropeptides also modulate inhibitory transmission but we did not detect this response likely due to their short-lasting effects that we did not include in our analyses (Crowley et al, 2016; Dumont and Williams, 2004).

Altogether, our data show that blocking CRFR1, NTR, and opioid receptors completely abolished post-synaptic activation-induced modulation of synaptic transmission in the rat ovBNST. This does not preclude that other stimulation patterns may trigger local synthesis and/or release of other neuromodulators (Puente et al, 2010) or of other neuropeptides expressed in ovBNST neurons (Woodhams et al, 1983). In addition, we focused on long-lasting changes in synaptic 
transmission but short-duration phenomena have also been reported (Puente et al, 2010). Unquestionably, numerous other neuropeptides, monoamines, or other molecules originating outside the ovBNST also robustly modulate synaptic transmission in this area (Dumont and Williams, 2004; Kash and Winder, 2006; Krawczyk et al, 2013; Krawczyk et al, 2011b; Li et al, 2012; McElligott and Winder, 2008; Shields et al, 2009). Nevertheless, the objective of this study was to determine whether local neuropeptidergic synaptic modulation was affected by chronic stress.

The neurophysiological mechanisms responsible for chronic stress-induced increase in anxiety-like behaviors are still largely unknown. Here CUS-facilitated l-DEI and NT was responsible for this effect, whereas the contribution of CRF was mitigated by stress. This is a novel observation considering that NT has been largely overlooked as a potential contributor in the pathological consequences of stress, compared to CRF (Saiz Ruiz et al, 1992). Alteration of NT function could be due to an increase in NT synthesis, release or receptor membrane expression, binding, or coupling. Under normal physiological conditions, NT increases inhibitory transmission by binding presynaptically to NTRs in the ovBNST (Krawczyk et al, 2013). NT increases excitability and firing rate in other brains areas but we did not detect post-synaptic changes in the membrane potential or membrane channels opening suggesting these were not altered in the ovBNST (Jassar et al, 1999; Xiao et al, 2014). Interestingly, the NTR antagonist reversibly depressed $\mathrm{GABA}_{\mathrm{A}}$-IPSC amplitude, in a seemingly inverse agonist way. The NTR2 exhibits constitutive activity on inositol phosphate production (Richard et al, 2001). Thus, the inverse agonist activity could occur through NTR2s that are also highly expressed in the BNST GABA neurons (Mazzone et al, 2016). However, it is still unknown whether the l-DEI is specific to or a combination of NTR1 and NTR2 activity and whether this is altered with CUS.

When we investigated mRNA expression, only Nts 1 mRNA, and not Nts or Ntsr2, was decreased in CUS rats compared to NS. Our findings corroborate other studies showing reduction of Ntsr 1 mRNA following maternal separation or CRF-overexpressing mice (Peeters et al, 2004; Toda et al, 2014). CUS decreasing Ntsr1 mRNA expression may not result in a reduction of the NTR1 receptor expression at the cellular membrane. A decrease in Ntsr1 mRNA could be due to an increase in mRNA stability or a compensatory mechanism to reduce increased NT activity. The latter explanation could indicate that NTR1 expression is actually increased with CUS and may be responsible for the changes in cell responses. Future studies investigating protein level expression is necessary to fully understand the neurophysiological changes occurring with CUS.

The CUS paradigm in this study utilizes variable and uncontrollable stress, where the animal does not habituate to the repeated stressors over time (Herman, 2013). As predicted from previous studies, we found that rats that underwent the CUS paradigm spent significantly less time in the open arms compared to their NS counterparts. Whether this behavior is 'adaptive' or 'maladaptive' is unclear. In the context of our EPM test, chronically stressed animals could be mounting an adaptive response to a reasonably imminent threat. Alternatively, their response could be interpreted as maladaptive as the animal limits their exploration and possibility of finding resources in the absence of an immediate threat. As the rats had no controllability over the stressors, it is impossible to distinguish whether they were mounting a contextually appropriate or inappropriate response.

Intra-ovBNST microinjections of a NTR antagonist did however robustly modify CUS rats behaviors in the EPM, tying the sensitized NT neurophysiological response in ovBNST neurons of stressed rats with their anxiety profile. NTR blockade reversed the anxiogenic effect of CUS without affecting normal EPM exploratory behavior displayed by NS rats. This is consistent with the fact that NT seems particularly important in mounting physiological and behavioral responses to face potentially extreme conditions (eg, store fat, seek rich and highly rewarding nutrients, and increase vigilance) (Deutch et al, 1987; Geisler et al, 2006; Krawczyk et al, 2013; Li et al, 2016; Luttinger et al, 1982). We also tested the effect of CUS on immobility in the FST but we did not find any changes in behavior previously reported (Bessa et al, 2009). This discrepancy may be due to the shorter duration of the stress paradigm although our data showed that NT in the ovBNST may not contribute to this behavior, regardless of the stress condition (Crestani et al, 2010).

Overall, these findings elucidate a clear role for NT in chronic stress although we cannot conclude exactly how NTinduced increase of GABA transmission in the ovBNST translates into anxiety-like behavior in the EPM. However, anatomical studies enable us to speculate how a NT-induced decrease of ovBNST activity could affect the HPA axis. The ovBNST has strong GABAergic outputs onto the fusiform nucleus of the BNST (fuBNST) that has direct inhibitory inputs onto the paraventricular nucleus of the hypothalamus (Dong et al, 2001). Lesion of the fuBNST attenuates HPA axis response suggesting it enhances PVN activity (Choi et al, 2007). As such, a NT-mediated reduction of ovBNST inhibition output to the fuBNST could promote HPA axis excitation and result in a decrease of EPM open arm exploration. Parallel to this, in the PVN, blocking NTR during stress counteracts the increase of plasma corticosterone levels (Geisler et al, 2006). In addition, decrease activity in ovBNST inhibitory projections could increase fear/anxiety (CeA), vigilance and arousal (substantia innominata), respiration (parabrachial nucleus), and defensive response (periaqueductal gray) (Dong et al, 2001). At this point however, we cannot discern the exact output of the ovBNST and whether it is affecting local or extrinsic circuitry.

The NT system in different brain areas could be working in concert to stimulate the HPA axis during stress conditions. Future studies should explore whether the magnitude of NT activation of the HPA axis could possibly correlate with maladaptive $v s$ adaptive behavior.

\section{FUNDING AND DISCLOSURE}

The authors declare no conflict of interest.

\section{ACKNOWLEDGMENTS}

CPN was funded by CIHR Vanier Graduate Scholarship (338319); APVS was funded by Fundação para a Ciência e 
Tecnologia (SFRH/BPD/52078/2013); ERH was funded by CIHR Postdoctoral Fellowship (MFE-123712); SA was funded by a Queen Elizabeth II Graduate Scholarship in Science and Technology; ÉCD was funded by the Canadian Institute of Health Research (MOP-25953).

\section{REFERENCES}

Bessa JM, Mesquita AR, Oliveira M, Pego JM, Cerqueira JJ, Palha JA et al (2009). A trans-dimensional approach to the behavioral aspects of depression. Front Behav Neurosci 3: 1.

Binder EB, Kinkead B, Owens MJ, Nemeroff CB (2001). Neurotensin and dopamine interactions. Pharmacol Rev 53: 453-486.

Cerqueira JJ, Mailliet F, Almeida OF, Jay TM, Sousa N (2007). The prefrontal cortex as a key target of the maladaptive response to stress. J Neurosci 27: 2781-2787.

Choi DC, Furay AR, Evanson NK, Ostrander MM, Ulrich-Lai YM, Herman JP (2007). Bed nucleus of the stria terminalis subregions differentially regulate hypothalamic-pituitary-adrenal axis activity: implications for the integration of limbic inputs. J Neurosci 27: 2025-2034.

Conrad KL, Louderback KM, Gessner CP, Winder DG (2011). Stress-induced alterations in anxiety-like behavior and adaptations in plasticity in the bed nucleus of the stria terminalis. Physiol Behav 104: 248-256.

Crestani CC, Alves FH, Correa FM, Guimaraes FS, Joca SR (2010). Acute reversible inactivation of the bed nucleus of stria terminalis induces antidepressant-like effect in the rat forced swimming test. Behav Brain Funct 6: 30.

Crowley NA, Bloodgood DW, Hardaway JA, Kendra AM, McCall JG, Al-Hasani R et al (2016). Dynorphin controls the gain of an amygdalar anxiety circuit. Cell Rep 14: 2774-2783.

Dabrowska J, Hazra R, Ahern TH, Guo JD, McDonald AJ, Mascagni $F$ et al (2011). Neuroanatomical evidence for reciprocal regulation of the corticotrophin-releasing factor and oxytocin systems in the hypothalamus and the bed nucleus of the stria terminalis of the rat: Implications for balancing stress and affect. Psychoneuroendocrinology 36: 1312-1326.

Dabrowska J, Hazra R, Guo JD, Li C, Dewitt S, Xu J et al (2013). Striatal-enriched protein tyrosine phosphatase-STEPs toward understanding chronic stress-induced activation of corticotrophin releasing factor neurons in the rat bed nucleus of the stria terminalis. Biol Psychiatry 74: 817-826.

Daniel SE, Rainnie DG (2016). Stress modulation of opposing circuits in the bed nucleus of the stria terminalis. Neuropsychopharmacology 41: 103-125.

Day HE, Curran EJ, Watson SJ Jr., Akil H (1999). Distinct neurochemical populations in the rat central nucleus of the amygdala and bed nucleus of the stria terminalis: evidence for their selective activation by interleukin-1beta. J Comp Neurol 413: 113-128.

Deppermann S, Storchak H, Fallgatter AJ, Ehlis AC (2014). Stressinduced neuroplasticity: (mal)adaptation to adverse life events in patients with PTSD-a critical overview. Neuroscience 283: 166-177.

Deutch AY, Bean AJ, Bissette G, Nemeroff CB, Robbins RJ, Roth RH (1987). Stress-induced alterations in neurotensin, somatostatin and corticotropin-releasing factor in mesotelencephalic dopamine system regions. Brain Res 417: 350-354.

Dong HW, Petrovich GD, Watts AG, Swanson LW (2001). Basic organization of projections from the oval and fusiform nuclei of the bed nuclei of the stria terminalis in adult rat brain. J Comp Neurol 436: 430-455.

Dumont EC, Williams JT (2004). Noradrenaline triggers GABAA inhibition of bed nucleus of the stria terminalis neurons projecting to the ventral tegmental area. J Neurosci 24: 8198-8204.
Geisler S, Berod A, Zahm DS, Rostene W (2006). Brain neurotensin, psychostimulants, and stress-emphasis on neuroanatomical substrates. Peptides 27: 2364-2384.

Glangetas C, Girard D, Groc L, Marsicano G, Chaouloff F, Georges F (2013). Stress switches cannabinoid type-1 (CB1) receptor-dependent plasticity from LTD to LTP in the bed nucleus of the stria terminalis. J Neurosci 33: 19657-19663.

Gosselin P, Laberge B (2003). [Etiological factors of generalized anxiety disorder]. Encephale 29(4 Pt 1): 351-361.

Hammack SE, Mania I, Rainnie DG (2007). Differential expression of intrinsic membrane currents in defined cell types of the anterolateral bed nucleus of the stria terminalis. J Neurophysiol 98: 638-656.

Hammen C, Kim EY, Eberhart NK, Brennan PA (2009). Chronic and acute stress and the prediction of major depression in women. Depress Anxiety 26: 718-723.

Herman JP (2013). Neural control of chronic stress adaptation. Front Behav Neurosci 7: 61.

Hubert GW, Muly EC (2014). Distribution of AMPA receptor subunit glur1 in the bed nucleus of the stria terminalis and effect of stress. Synapse 68: 194-201.

Iremonger KJ, Bains JS (2009). Retrograde opioid signaling regulates glutamatergic transmission in the hypothalamus. J Neurosci 29: 7349-7358.

Jassar BS, Harris KH, Ostashewski PM, Jhamandas JH (1999). Ionic mechanisms of action of neurotensin in acutely dissociated neurons from the diagonal band of Broca of the rat. J Neurophysiol 81: 234-246.

Ju G, Han ZS (1989a). Coexistence of corticotropin releasing factor and neurotensin within oval nucleus neurons in the bed nuclei of the stria terminalis in the rat. Neurosci Lett 99: 246-250.

Ju G, Swanson LW, Simerly RB (1989b). Studies on the cellular architecture of the bed nuclei of the stria terminalis in the rat: II. Chemoarchitecture. J Comp Neurol 280: 603-621.

Kash TL, Winder DG (2006). Neuropeptide Y and corticotropinreleasing factor bi-directionally modulate inhibitory synaptic transmission in the bed nucleus of the stria terminalis. Neuropharmacology 51: 1013-1022.

Kim SY, Adhikari A, Lee SY, Marshel JH, Kim CK, Mallory CS et al (2013). Diverging neural pathways assemble a behavioural state from separable features in anxiety. Nature 496: 219-223.

Krawczyk M, Georges F, Sharma R, Mason X, Berthet A, Bezard E et al (2011a). Double-dissociation of the catecholaminergic modulation of synaptic transmission in the oval bed nucleus of the stria terminalis. J Neurophysiol 105: 145-153.

Krawczyk M, Mason X, DeBacker J, Sharma R, Normandeau CP, Hawken ER et al (2013). D1 dopamine receptor-mediated LTP at GABA synapses encodes motivation to self-administer cocaine in rats. J Neurosci 33: 11960-11971.

Krawczyk M, Sharma R, Mason X, Debacker J, Jones AA, Dumont EC (2011b). A switch in the neuromodulatory effects of dopamine in the oval bed nucleus of the stria terminalis associated with cocaine self-administration in rats. J Neurosci 31: 8928-8935.

Larriva-Sahd J (2006). Histological and cytological study of the bed nuclei of the stria terminalis in adult rat. II. Oval nucleus: extrinsic inputs, cell types, neuropil, and neuronal modules. I Comp Neurol 497: 772-807.

Laszlo K, Toth K, Kertes E, Peczely L, Ollmann T, Lenard L (2010). Effects of neurotensin in amygdaloid spatial learning mechanisms. Behav Brain Res 210: 280-283.

Lezak KR, Roman CW, Braas KM, Schutz KC, Falls WA, Schulkin J et al (2014). Regulation of bed nucleus of the stria terminalis PACAP expression by stress and corticosterone. J Mol Neurosci 54: 477-484.

Li C, Pleil KE, Stamatakis AM, Busan S, Vong L, Lowell BB et al (2012). Presynaptic inhibition of gamma-aminobutyric acid release in the bed nucleus of the stria terminalis by kappa opioid receptor signaling. Biol psychiatry 71: 725-732. 
Li J, Song J, Zaytseva YY, Liu Y, Rychahou P, Jiang K et al (2016). An obligatory role for neurotensin in high-fat-diet-induced obesity. Nature 533: 411-415.

Ludwig M, Pittman QJ (2003). Talking back: dendritic neurotransmitter release. Trends Neurosci 26: 255-261.

Luttinger D, King RA, Sheppard D, Strupp J, Nemeroff CB, Prange AJ Jr. (1982). The effect of neurotensin on food consumption in the rat. Eur J Pharmacol 81: 499-503.

Mazzone CM, Pati D, Michaelides M, DiBerto J, Fox JH, Tipton G et al (2016). Acute engagement of Gq-mediated signaling in the bed nucleus of the stria terminalis induces anxiety-like behavior. Mol Psychiatry (epub ahead of print).

McElligott ZA, Klug JR, Nobis WP, Patel S, Grueter BA, Kash TL et al (2010). Distinct forms of Gq-receptor-dependent plasticity of excitatory transmission in the BNST are differentially affected by stress. Proc Natl Acad Sci USA 107: 2271-2276.

McElligott ZA, Winder DG (2008). Alpha1-adrenergic receptorinduced heterosynaptic long-term depression in the bed nucleus of the stria terminalis is disrupted in mouse models of affective disorders. Neuropsychopharmacology 33: 2313-2323.

Peeters PJ, Fierens FL, van den Wyngaert I, Goehlmann HW, Swagemakers SM, Kass SU et al (2004). Gene expression profiles highlight adaptive brain mechanisms in corticotropin releasing factor overexpressing mice. Brain Res Mol Brain Res 129: 135-150.

Pego JM, Morgado P, Pinto LG, Cerqueira JJ, Almeida OF, Sousa N (2008). Dissociation of the morphological correlates of stress-induced anxiety and fear. Eur J Neurosci 27: 1503-1516.

Poulin JF, Arbour D, Laforest S, Drolet G (2009). Neuroanatomical characterization of endogenous opioids in the bed nucleus of the stria terminalis. Prog Neuropsychopharmacol Biol Psychiatry 33: 1356-1365.

Puente N, Elezgarai I, Lafourcade M, Reguero L, Marsicano G, Georges F et al (2010). Localization and function of the cannabinoid $\mathrm{CB} 1$ receptor in the anterolateral bed nucleus of the stria terminalis. PloS one 5: e8869.

Richard F, Barroso S, Martinez J, Labbe-Jullie C, Kitabgi P (2001). Agonism, inverse agonism, and neutral antagonism at the constitutively active human neurotensin receptor 2. Mol Pharmacol 60: 1392-1398.

Saiz Ruiz J, Carrasco Perera JL, Hernanz A (1992). Plasma neuropeptides in affective and anxiety disorders. Arch Neurobiol (Madr) 55: 1-5.

Shields AD, Wang Q, Winder DG (2009). alpha2A-adrenergic receptors heterosynaptically regulate glutamatergic transmission in the bed nucleus of the stria terminalis. Neuroscience 163: 339-351.

Toda H, Boku S, Nakagawa S, Inoue T, Kato A, Takamura N et al (2014). Maternal separation enhances conditioned fear and decreases the mRNA levels of the neurotensin receptor 1 gene with hypermethylation of this gene in the rat amygdala. PLoS ONE 9: e97421.

Vyas A, Bernal S, Chattarji S (2003). Effects of chronic stress on dendritic arborization in the central and extended amygdala. Brain Res 965: 290-294.

Walker DL, Miles LA, Davis M (2009). Selective participation of the bed nucleus of the stria terminalis and CRF in sustained anxietylike versus phasic fear-like responses. Prog Neuropsychopharmacol Biol Psychiatry 33: 1291-1308.

Woodhams PL, Roberts GW, Polak JM, Crow TJ (1983). Distribution of neuropeptides in the limbic system of the rat: the bed nucleus of the stria terminalis, septum and preoptic area. Neuroscience 8: 677-703.

Xiao Z, Cilz NI, Kurada L, Hu B, Yang C, Wada E et al (2014). Activation of neurotensin receptor 1 facilitates neuronal excitability and spatial learning and memory in the entorhinal cortex: beneficial actions in an Alzheimer's disease model. J Neurosci 34: 7027-7042.

Supplementary Information accompanies the paper on the Neuropsychopharmacology website (http://www.nature.com/npp) 